\title{
(Un-)Verfügbarkeit. Bonaventuras Stigmata
}

Kiening, Christian

Posted at the Zurich Open Repository and Archive, University of Zurich ZORA URL: https://doi.org/10.5167/uzh-91497

Book Section

Published Version

Originally published at:

Kiening, Christian (2012). (Un-)Verfügbarkeit. Bonaventuras Stigmata. In: Kasten, Ingrid. UnVerfügbarkeit. Berlin: Akademie-Verlag, 25-35. 


\section{Christian Kiening}

\section{(Un-)Verfügbarkeit}

\section{Bonaventuras Stigmata ${ }^{1}$}

The article traces the construction of the legend surrounding the mystery of Francis of Assisi's stigmata, which are said to have appeared two years before his death. Central to this analysis, which is underpinned by sign and media theory, is the Legenda maior, a description of the life of Francis, written by Bonaventura four decades later on the basis of older testimonies and legends. The focus is on those strategies which allow the change of events between mediation and immediacy, in that they, on the one hand, are dominated by a concrete, spatial framework and, on the other, by moments of that which is unverfügbar.

\section{I}

Von allem Anfang an war das Ereignis der Stigmatisierung, das auf das Jahr 1224 datiert wird, zwei Jahre vor dem Tod des Franziskus, umstritten. ${ }^{2}$ Zwar hatte Elias von Cortona, der Nachfolger des Franziskus in der Leitung des Minoritenordens, unmittelbar nach dessen Tod einen Brief an die Provinzialminister des Ordens geschrieben, in dem er im Ton des Evangelisten Lukas angesichts der Geburt Christi „eine große Freude und die Neuheit eines Wunders“ verkündete (gaudium magnum et miraculi novitatem) und einige Details über die Nägel und die Seitenwunde mitteilte. Auch hatte Thomas von Celano, als er 1228/29 nach der Kanonisierung in offiziellem päpstlichen Auftrag die erste Lebensbeschreibung des Franziskus verfasste, weitere Komponenten hinzugefügt, die für die Folgezeit Bedeutung haben werden: die Erscheinung eines Seraphs, der zwischen seinen sechs Flügeln die Gestalt eines Gekreuzigten zeigt, und das Öffentlichwerden der Stigmata. Doch die eigentliche Diskussion der Stigmata stand erst an ihrem Anfang. Nicht nur gab es Kritik von außerhalb: Weltkleriker und Mitglieder anderer Ordensgemeinschaften hatten ihre eigenen Interessen und waren nur bedingt willig, sich dem Glauben an das unerhörte Ursprungsereignis an-zuschließen. Auch innerhalb des Franziskanerordens waren die Meinungen geteilt. Die frühen Gefährten des Franziskus hingen anscheinend eher der schon von Thomas von Celano formulierten, aber nicht befolgten Maxime an, es sei besser, dem wahren Grund des „Ehrfurcht

1 Eine ausführlichere Version dieses Beitrags findet sich in dem Buch Urszenen des Medialen von Christian Kiening/Ulrich Johannes Beil (2012).

2 Eine genaue Rekonstruktion der innerfranziskanischen Diskurse bietet zuletzt (mit allerdings umstrittenen Schlussfolgerungen) Bösch 2005; vgl. auch Adnès 1990; Schmucki 1991; Frugoni 1993; Bouflet 1996; Daxelmüller 2001; Menke/Vinken 2004. 
gebietenden Mysteriums“ nicht zu genau nachzuforschen. Diejenigen hingegen, welche die Stigmata ins Zentrum der institutionellen Stabilisierung des Ordens stellen wollten, hatten sich auch auf den Treibsand der Authentifizierung einzulassen.

Das geschah vor allem in den 1250er Jahren, als es für die Franziskaner darum ging, den Umgang mit der eigenen wachsenden Geschichtlichkeit zu klären. Während die Spiritualen an einem radikalen Armutsideal festhielten, trat die Kommunität für Anpassungen an die gegenwärtigen Bedürfnisse ein; sie wollte den Orden eher im Zentrum der sozialen Gefüge der Zeit als an deren Rand verankert wissen. ${ }^{3}$ Dabei scheint auch den Stigmata eine neue Rolle zugewachsen zu sein: Als unhintergehbares Zeichen einer durch Christus selbst approbierten Lebensweise wurden sie zu einem starken Argument im Diskurs. ${ }^{4}$ Papst Alexander IV. etwa, ein entschiedener Protektor des Minoritenordens, war ein Verehrer des Bergs La Verna und sah sich selbst als Augenzeugen der Wundmale. Er ließ die Berichte über die Stigmatisierung, ja sogar die Aussagen über die Beschaffenheit der Wundmale offiziell anerkennen und drohte Leugnern mit Exkommunikation und Amtsenthebung. Es entstand ein Diskussionszusammenhang, in dem der Raum zwischen den Extremen ausgelotet wurde: hier das selbstbestimmte, imaginative, asketische Virtuosentum des Heiligen, dort seine fremdbestimmte, real-körperliche, mirakulöse Apotheose.

Das ist die Situation, in der Bonaventura die Bühne betritt. Einerseits eine neue Geltung der frühen Augenzeugen und der archaischen Überlieferung, andererseits eine Tendenz zur systematischen Rekonstruktion. Einerseits ein fortwirkendes Gewirr der Stimmen, andererseits die verstärkte Bemühung um eine ,unité de doctrine', die Sehnsucht, aus allen vorhandenen Legenden, wie das Generalkapitel in Rom 1257 formulierte, ,eine gute“ zusammenzustellen, eine gültige, an der sich auch die Liturgie ausrichten konnte. Bonaventura, der diese Aufgabe übernimmt, ist ein Vertreter der zweiten Generation. Selbst beim Tod des Franziskus fünf Jahre alt, kennt er immerhin einige der Gefährten und kann von der Präsenz der Augenzeugen zehren:

Um das Leben des Heiligen, das ich der Nachwelt überliefern soll, wie es tatsächlich verlaufen ist, sicherer und klarer vor Augen zu haben, habe ich die Stätte seiner Geburt, seines Lebens und seines Heimgangs aufgesucht und mich mit seinen vertrauten Gefährten, die noch am Leben sind, eingehend unterhalten, vor allem mit denen, die am besten seine Heiligkeit erkannt und nachgeahmt haben $(L M, \text { Prol. } 4)^{5}$

3 Vgl. Dalarun 1999; Melville/Oberste 1999.

4 Vgl. Bösch 2005, S. 109-117.

5 Lateinische Texte: Bonaventura, Legenda maior [abgekürzt zitiert LM] (1926-1941); ders., Legenda minor (1926-1941); Nachdruck: Enrico Menestò e Stefano Brufani et al. (1995). Deutsche Übersetzung: Clasen 1962. 
Auf der Ebene der Fakten bietet Bonaventura in seiner Legenda maior kaum Neues. Er übernimmt das Meiste von den Vorgängern. Streckenweise, bei der Frühgeschichte oder auch den Mirakeln, schreibt er die Texte des Thomas von Celano regelrecht aus. Doch bereinigt er Uneinheitlichkeiten und lässt Zweifelhaftes aus. Er macht die eigene Darstellung zu einem Gesamtkunstwerk, das die früheren Legenden ablösen sollte und einen autoritativen Bezugspunkt für die spätere Franziskushistoriographie bot.

Bonaventuras Text folgt eingangs der Chronologie. Der Hauptteil der 15 Kapitel indes zielt auf eine thematische Ordnung nach „,sachlichen Zusammenhängen“ (ordo aptae iuncturae). Arrangement und Konzeption, Stringenz und Balance der Erzählung sind dementsprechend höchst reflektiert. Es entsteht ein übergreifender Deutungsrahmen, der sich von der Selbstwahrnehmung des Franziskus über die Perspektive seiner Umgebung bis hin zur Situation des schreibenden Biographen und seiner Leserschaft erstreckt. Es deutet sich eine Folge von Wegen an, die von der via purgativa über die via illuminativa zur via unitiva führen - und in den Stigmata gipfeln. ${ }^{6}$

Wie zentral diese für die Legenda sind, zeigt sich schon im Prolog, der alles an Franziskusmetaphern anhäuft, was die älteren Viten boten. ${ }^{7}$ Der poverello erscheint in einer Linie mit den Propheten Elias und Johannes Baptista. Er wird vor dem Hintergrund des Buches Ecclesiasticus mit dem Morgenstern und dem Regenbogen verglichen. Er gilt aber auch als Verkörperung eines in der Johannesapokalypse geweissagten Bildes (similitudo): als Engel des sechsten Siegels, der „vom Aufgang der Sonne aufsteigt und das Zeichen des lebendigen Gottes trägt." ${ }^{8}$ Bonaventura verknüpft diesen Gedanken mit den Stigmata (signum Dei vivi) und rückt diese damit ins Zentrum seiner Theologie - einer Theologie des Zeichens und des Körpers, die alle Möglichkeiten von Evidenz und Signifikanz ausschöpft: geheimnisvolle Präsenzen und machtvolle Momente, aber auch Analogien, Ähnlichkeiten, Verweise. Alles scheint hier um die Frage zu kreisen, wie der Mensch über das eine Medium Christus, seine Wiederverkörperung in Franziskus und die Medien der Vergegenwärtigung (Vision, Bild, Schrift) zum Heil gelangen könne; aber auch um die Frage, wie ein Ereignis in seiner absoluten Singularität herausgestellt, zugleich aber in seiner Authentizität diskursiv gesichert werden kann.

Deshalb werden die Lesenden schrittweise auf den La Verna geführt, wo sie beinahe aus der Innenperspektive die Erscheinung des Seraphs vor Franziskus wahrnehmen. Sie werden dann aber auch mit der Diskussion konfrontiert, was vom Ereignis an welche Augen, Ohren und Hände gelangt. Die direkt auf den Abstieg vom La Verna folgenden Wunderberichte setzen auf eine unmittelbare Übertragung der Heilsenergie - von einem armen Mann heißt es: „kaum fühlte er sich von der Hand des Heiligen, die die glühende Kohle des Seraphs trug, angerührt, da spürte er keine Kälte mehr, und er empfand innerlich und äußerlich solche Wärme,

6 Vgl. Bougerol 1984, S. 36-46.

7 Vgl. Brooke 2006, S. 255.

8 Zu Bonaventuras Schwerpunkt auf dem apokalyptischen Franziskus vgl. Flood 2005. 
als ob eine Feuersglut aus einem Ofen ihm entgegenstrahle" ( $L M$ II,13,7). Der sich daran anschließende Passus hingegen führt die Fälle auf, in denen die Wundmale geschaut wurden. Hier dominiert die Bemühung, genau zu belegen, welche Formen (Schwören auf das Evangeliar, Zeugnisse in Wort und Schrift, Predigt) und welche Personen (Brüder, Schwestern, Kardinäle, Papst) für die Authentisierung einstehen können. Die Quantitäten, die Zahl der Zeugen, das Ausmessen der Wunde, gewinnen an Bedeutung.

Charakteristisch ist so im Ganzen ein Changieren zwischen Vermittlung und Unmittelbarkeit. Einerseits gibt es konkrete räumliche und zeitliche Rahmenbedingungen: der Berg La Verna, die Fastenzeit, das Fest der Kreuzerhöhung. Sie verankern die Stigmatisierung in der christlichen Tradition und im christlichen Jahreszyklus. Andererseits treten Momente des Unverfügbaren heraus: der blitzschnelle Flug des Seraphs, der geheimnisvolle Charakter der Erscheinung, die Wunder, durch die ,die verborgene und staunenswerte Kraft der Wundmale im Glanz der Zeichen offenkundig“" wird. ${ }^{9}$ Der „Glanz der Zeichen“ (claritas signorum) - das ist das Stichwort, unter das sich die ganze Episode stellen lässt: Als zeichenhaft sollen die Ereignisse begriffen werden, also als verweisend, bezogen auf etwas, mit dem sie nicht identisch sind. Zugleich sollen diese Zeichen durchscheinend sein für das Bezeichnete, nicht getrübt durch die Brechung eines Zeichenträgers. Mehr als bloße Anzeiger, sollen sie die Fülle des Heils selbst ein Stück weit verkörpern, die Christusförmigkeit des Franziskus zur Erfüllung bringen.

In diesen Zeichen verwirklicht sich auch die Idee der Transsubstantiation, an der Bonaventura in seinem Frühwerk, dem Sentenzenkommentar, gearbeitet hatte. Sie verwirklicht sich in einer konkreten Gestalt - nicht allerdings im Sinne geläufiger Eucharistiemirakel oder Hostienvisionen. ${ }^{10}$ In Franziskus verwandelt sich die Hostie nicht einfach momenthaft-visionär in den blutenden Leib des Jesuskindes. In ihm ist das Urbild selbst dauerhaft da. Oder anders gesagt: ist die Verehrung Christi, seines Leibes und seiner Spuren, von einer Anverwandlung an Christus im Sinne einer Neuverkörperung kaum zu trennen. Franziskus ist die ,lebende Ikone', in der ,künstliche' und ,natürliche', bildliche und körperliche Vermittlungsformen zusammentreffen. ${ }^{11}$

Dabei war aber der Eindruck abzuwehren, Franziskus habe die Anverwandlung aus eigenen Stücken betrieben: voluit crucifigi, ,er wollte gekreuzigt werden“, heißt es bei Bonaventura, doch die Realisierung dieses Willens steht nicht in der Macht des virtuosen Asketen, sie bedarf einer Verwandlung durch das, an das er sich anverwandelt. Deshalb war es der franziskanischen Tradition wichtig, dass der Ordensgründer als Zeichen seiner humilitas die Stigmata geheim zu halten versuchte, dass diese Geheimhaltung aber nur bedingt funktionierte. ${ }^{12}$ Und deshalb finden sich in der Legenda an zentralen Stellen Hinweise auf die göttliche Steuerung des

9 LM XIII,5: ut illorum occulta et mira vis stigmatum manifesta pateret claritate signorum.

10 Vgl. Browe 1938, 2003.

11 Belting 2006, S. 30 und 23.

12 In diesem Zusammenhang wird auch die Kutte des Franziskus eine wichtige Rolle spielen; vgl. Kapustka 2010. 
Geschehens: Die divina providentia bringt Franziskus zum Aufstieg auf den Berg; ein divinum oraculum rät ihm, die Bibel selbst als prophetisches Mittel zu benutzen; eine göttliche Offenbarung (Domino revelante) ermöglicht ihm, die Erscheinung des Seraphs zu verstehen.

Vor allem das divinatorische Aufschlagen der Evangelien zeigt an, dass sich auf dem La Verna die Beziehung von Franziskus zu Christus auf höheres Geheiß hin intensivieren wird. Zugleich stiftet es einen Bezug zur Tradition: Augustinus hatte bekanntlich seine endgültige conversio unter dem Eindruck des gehörten tolle, lege vollzogen, das ihn auf den Römerbrief des Paulus führte (Conf. 8,12,29). Franziskus selbst hatte, nach dem Bericht der Biographen, die Anfänge seiner Bewegung an ein Buchorakel geknüpft: 1208 begab er sich mit einem seiner ersten Anhänger, Bernhard von Quintavalle, zur Kirche San Nicolò in Assisi, um herauszufinden, was der rechte Lebensweg sei - dreimal stieß er beim Öffnen des Missales auf Stellen aus Matthäus und Lukas, welche die eigene Idee von Armut und Weltferne bestätigten. ${ }^{13}$ Mit dem neuerlichen Buchorakel auf dem La Verna rundet sich somit seine Geschichte ebenso wie die des Ordens. Anders als eineinhalb Jahrzehnte zuvor führt nun das Aufschlagen des Evangeliars dreimal auf die Passionsgeschichte: $\mathrm{Da}$,erkannte der von Gott erfüllte Mann, er müsse, bevor er aus dieser Welt scheide, Christus in der Bedrängnis und in dem schmerzvollen Leiden ähnlich werden, wie er ihn zeitlebens in seinem Handeln nachgeahmt hatte. ${ }^{\text {“14 }}$ Der Unterschied des gegenwärtigen Moments zur vorausgehenden Lebensform liegt zwischen imitatio und conformitas: Die eine verweist auf einen bewusst betriebenen Akt, die andere auf ein unverfügbares Geschehen. Entscheidend ist ihr Zusammentreffen, in dem subjektive und objektive Faktoren eine dynamische Einheit bilden.

\section{III}

Der Prozess des Ähnlichwerdens vollzieht sich bei Bonaventura oszillierend zwischen Innen und Außen, Vision und Realisation, Urbild und Nachbildung, Sprache und Wirklichkeit, Schriftzitat und -umsetzung. Schon am Beginn des Kapitels wird die Vermittlung zwischen Irdischem und Überirdischem im traditionellen Bild der Leiter gefasst, genauer: der Jakobsleiter aus dem Buch Genesis $(28,12)$, auf der der Patriarch ,wie die Himmelsgeister [...] zu Gott empor oder zum Nächsten hernieder" stieg (LM II,13,1). Der träumende Jakob wird zum Modell des Visionärs Franziskus, der, wie Moses auf den Sinai, auf den La Verna steigt, um dem Göttlichen nahe zu sein und sich im Geiste über die Welt zu erheben. Das Szenario erhält aber sogleich neutestamentlich-christologische Akzente; zitathaft klingen die Aussendungsrede Jesu (Lc 10,11) und dessen Verklärung auf dem Berg Tabor (Mt 17,1)

13 Vgl. Feld 1994, S. 145-150.

14 LM XIII, 2: intellexit vir Deo plenus, quod sicut Christum fuerat imitatus in actibus vitae, sic conformis ei esse deberet in afflictionibus et doloribus passionis, antequam ex hoc mundo transiret (vgl. Io 13,1). 
an. Das Muster von Ankündigung und Erfüllung rückt Franziskus in ein Verhältnis zu Christus, das analog ist zu dessen Verhältnis zu den vorangegangenen Propheten. Zugleich aber vermehrt Bonaventura die Referenzen: Franziskus bezieht sich außer auf Christus auch auf dessen verschiedene Vorgänger und auf den Engel der Endzeit. Er wird zu einer Figur, in der alle Zeiten sich zu schneiden scheinen. Ein universales Signifikat, auf das sich unendlich viele Signifikanten beziehen können. Ein Mittler, in dem je neu Vergangenes in Gegenwärtiges sich verwandelt, Sprache in Körperlichkeit umschlägt, aber auch die Medialität der Verkörperung - in Form der Vision, des Zeichens, des Bildes - aufscheint.

Sorgfältig führt Bonaventura auf den Höhepunkt hin: die Isolation von der Welt, der Aufstieg auf den Berg, das Sichversenken in die Passion, die Sehnsucht nach dem Martyrium - all das bereitet den Weg für jene entscheidenden zwei Ereignisse, in denen das Geschehen kulminiert: das Erscheinen des Seraphs und die Übertragung der Stigmata. Seraphisch ist das Begehren des Franziskus, und ein Seraph ist es, der ihm begegnet. Auch dies steht vor alttestamentlichem Hintergrund. Franziskus erlebt, was Jesaja erlebte, als er zum Propheten berufen wurde:

In dem Jahr, als der König Usija starb, sah ich den Herrn sitzen auf einem hohen und erhabenen
Thron und sein Saum füllte den Tempel. Serafim standen über ihm; ein jeder hatte sechs Flügel:
Mit zweien deckten sie ihr Antlitz, mit zweien deckten sie ihre Füße und mit zweien flogen sie
(Jes 6,1-3).

Die Änderung, die Bonaventura vornimmt, ist unscheinbar, aber gewichtig: „Zwei Flügel erhoben sich über dem Haupt, zwei waren zum Flug ausgebreitet, zwei verhüllten den ganzen Körper" ${ }^{15}$ Damit ist das Verhältnis von enthüllten und verhüllten Teilen vertauscht: Der in Jesajas Vision anscheinend sichtbare Körper bleibt für Franziskus unsichtbar, statt dessen aber zeigt sich ein anderer Körper - derjenige des Gekreuzigten. Wieder wird also das Szenario auf die Passion Christi bezogen, doch nun nicht im Sinne eines sprachlichen Ineinanderblendens von Altem und Neuem Testament, sondern als eigentümliche, ja skandalöse Verschmelzung des makellosen Engels mit dem gepeinigten Menschensohn, des Unsterblichen mit dem Sterblichen.

Wie ist das Gefüge aus Seraph und Crucifixus zu denken? „In der Gestalt eines Seraphs“ (sub specie Seraph) begegnet Christus Franziskus - ein unüberhörbarer Anklang an die Formel der Transsubstantiation: „in der Gestalt des Brotes“ (sub specie panis). ${ }^{16}$ Wo genau zeigt sich der Gekreuzigte? „Zwischen den Flügeln“, aber zwischen welchem der Flügelpaare? Bonaventura hat ganz offensichtlich kein Interesse, auf diese und andere Fragen Antworten zu liefern. Vielmehr eröffnet er durch seine Terminologie zusätzliche Interpretationsspielräume. Der Engel trägt ein Bild des Gekreuzigten: effigies hominis crucifixi. Franziskus begegnet also nicht Christus selbst, sondern einer Repräsentation - die aber mit dem schillernden

15 Ebd.: Duae alae super caput ipsius elevabantur, duae ad volandum extendebantur, duae vero totum velabant corpus.

16 Vgl. Davidson 2009. 
Begriff effigies belegt wird. Der Begriff kann generell ein materielles Bild im Unterschied zu einem imaginierten, aber auch das wahre Antlitz Christi, die Totenmaske, im späten Mittelalter überdies die Nachbildung des toten Körpers des Königs bezeichnen - er transportiert jedenfalls Momente einer magischen Realpräsenz. ${ }^{17}$ Oder auch: Momente einer Kopie, die keine ist. Während bei Thomas von Celano (1. Vita) der poverello einen Mann über sich schweben sieht, der einem Seraph ähnlich ist (quasi Seraphim), trennt Bonaventura zunächst deutlicher zwischen Seraph und Crucifixus - nur um im nächsten Schritt eine subtile Übertragung zu vollziehen. Das Bild, so die Suggestion, sei mehr als ein bloßes Bild oder Abbild. Es rückt in die Nähe des Abdrucks oder des Ebenbilds. Es hinterlässt einen Eindruck. Es blickt Franziskus an (cernebat se conspici). Es schürt ein wunderbares Feuer im Herzen. Und es macht schließlich Platz für eine weitere effigies - diejenige der Wundmale (signa), die dem Leibe, nicht weniger wunderbar als die Vision selbst, eingeprägt worden sei (impressit). Die Vermittlung der Christuserscheinung durch den Engel wird somit in die Unvermitteltheit der sich zeigenden Stigmata überführt. Doch zwischen beiden steht eine nicht-bildliche Offenbarung Gottes (Domino revelante). Sie ist es, die Franziskus den Sinn der Erscheinung enthüllt: „damit er schon jetzt wisse (praenosset), nicht der Martertod des Leibes, sondern die Glut des Geistes müsse ihn als Freund Christi ganz zum Bild des gekreuzigten Christus umgestalten." 18

Das aber heißt auch: Die Lesenden erleben nicht einfach einen vom Gnadengeschehen ergriffenen Protagonisten, sondern einen, dessen Wahrnehmung zwischen einem äußeren und einem inneren Sehen schwankt, der sich von der Immersion zur Reflexion bewegt, der vom Sehen über Empfindung und Staunen zum Begreifen gelangt. In dem Moment, in dem er versteht (intellexit), verschwindet die Erscheinung (disparens visio) und lässt zugleich ihre Spuren zurück: innerliche (reliquit ardorem) und äußerliche (impressit effigiem). Auf diese Weise werden äußerste Geistigkeit und äußerste Körperlichkeit enggeführt. Die Vision erscheint zugleich als imaginative und korporale. Die Stigmatisierung wird zugleich als Handlungswie als Wahrnehmungs- und Reflexionsgeschehen erzählt. Dass Bonaventura nun für dasjenige, was mit Franziskus geschieht, den gleichen Terminus effigies benutzt, den er zuvor für das Bild des Gekreuzigten verwendet hatte, stiftet eine Beziehung zwischen dem ersten, visionären und dem zweiten, körperlichen Eindruck. Sie wird unterstrichen durch den Hinweis, die sichtbar werdenden Stigmata würden den Zeichen entsprechen, ,wie er sie soeben an jenem Bild des Gekreuzigten geschaut hatte". ${ }^{19}$

Erst nachdem er so ein komplexes Netz an wechselseitigen Verweisen gespannt hat, beschreibt Bonaventura die Stigmata selbst - die durchbohrten Hände und Füße, an denen sich noch die schwarzen Köpfe und die umgebogenen Spitzen der Nägel zeigen, aus dem Fleisch herauswachsend und über dieses hinausragend, die

17 Vgl. Marek 2008.

18 LM XIII,5: ut amicus praenossit, se non per martyrium carnis, sed per incendium mentis totum in Christi crucifixi similitudinem transformandum.

19 LM XIII,5: quemadmodum paulo ante in effigie illa viri crucifixi conspexerat. 
klaffende Seitenwunde, wie „,von einer Lanze durchstochen; aus ihr floß oft Blut hervor, so daß sein Habit und seine Hose davon benetzt wurden“ ${ }^{20}$ Das sind genau die Worte, die schon Thomas von Celano in seiner ersten Vita gebraucht hatte. Wie eine authentische Spolie aus der Frühzeit der Ordensgeschichte wirken diese Sätze, tausend Mal gelesen und wiederholt, quasi zur Formel geworden für die franziskanische Kommunität - und für Momente auch bei Bonaventura von keiner Kommentatorenstimme unterbrochen.

\section{IV}

Ansonsten aber ist es gerade die Vielfalt der Stimmen und Perspektiven, die die Legenda prägt. Mal erlebt man Franziskus von außen, mal von innen. Mal werden Ereignisse, mal Gefühle und Wahrnehmungen, mal Deutungen mitgeteilt. Mal gibt es greifbare Sachverhalte, mal metaphorische Eigendynamiken. Die Begrifflichkeit zeigt sich infiziert von der Tradition, der alt- und der neutestamentlichen, der patristischen und der scholastischen, aber auch der zeitgenössischen Theologie und Mystik. Sie dient der diskursiven Verortung des Geschehens und sprengt zugleich immer wieder das Diskursive, indem sie Präsenzen produziert. Bonaventuras Systematisierung ist denn auch nicht zu trennen von einer Emphatisierung durch Ausrufe, Anreden, Wiederholungen, Echoeffekte, semantische Aufladungen. Bei aller reflexiven Durchdringung ist das Gewebe der Begriffe nicht darauf angelegt, das Ereignis selbst zu rationalisieren. Diesem soll sein geheimnisvoller Charakter bleiben.

Enthüllen und Verhüllen bleiben in der franziskanischen Medialität ebenso aneinander gebunden wie die Rolle des Körpers als Quelle und Träger des Sinns, als heilserfülltes göttliches und unzureichendes irdisches Medium. ${ }^{21}$ Es ergibt sich so eine Funktion der Stigmata als Beweisstücke (für die Gültigkeit der franziskanischen Regel), als Zeichen (eines übernatürlichen Geschehens), als Siegel (eines singulären Ereignisses) und als Male (einer realen Übertragung). Und es ergibt sich eine Christusförmigkeit zwischen äußerem und innerem Bild, zwischen realer und bildhafter conformitas, zwischen imago, visio, effigies und impressio, auch zwischen einem transsubstantiierten und einem transfigurierten Körper: Im Tode nämlich, mit dem Bonaventura die Vita beschließt, sind die Wundmale deutlicher denn je sichtbar, schwarz die Nägel, rot wie eine blühende Rose die Seitenwunde, doch nunmehr an einem schneeweißen, strahlend schönen, verklärten Körper: „Seine Glieder fühlten sich so weich und geschmeidig an, als hätten sie die Zartheit des Kindesalters zurückerlangt; andern erschienen sie mit den sichtbaren Zeichen der Sündenlosigkeit geziert“ $(L M X V, 3)$.

20 Ebd.: quasi lancea transfixum, rubra cicatrice obductum erat, quod saepe sanguinem sacrum effundens, tunicam et femoralia respergebat.

21 Pavlick 2007. 
Die Christusförmigkeit des Franziskus legt bei Bonaventura keine totale Identität nahe, wohl aber eine maximale Nähe zum christologischen Ereignis. Ihre eigene Ereignishaftigkeit gipfelt sprachlich erst mit dem Abstieg vom La Verna, der wiederum an den des Moses vom Sinai erinnert. Franziskus habe das Bild des Gekreuzigten an sich getragen, ,das nicht Künstlerhand auf Tafeln aus Stein oder Holz gemeißelt, sondern der Finger des lebendigen Gottes den Gliedern seines Leibes eingeprägt hatte“. ${ }^{22}$ Der Satz bezieht sich auf die berühmte Szene, in der JHWH Moses die beiden Tafeln des Zeugnisses übergibt: „Tafeln aus Stein, beschrieben vom Finger Gottes" (Gen 31,18). Er setzt aber auch die paulinische Umcodierung dieser mosaischen Schrift voraus, die ja gerade darauf hinausgelaufen war, nicht die Schrift auf Stein, sondern die des Herzens ermögliche eine unmittelbare Kommunikation (2 Kor 3,3). Diese Vorstellung erfährt nun eine weitere Umcodierung: vom Herz auf den Körper (in carneis membris). Das heißt: In Franziskus konvergieren die mosaische Tradition einer exklusiven göttlichen Schrift und die paulinische einer universalen, durch Christus ermöglichten Kommunikation der Herzen. Der Ordensgründer erweist sich nicht einfach als Träger von Zeichen, sondern als Medium der göttlichen Selbstmitteilung, in dem sich die christologische Medialität auf eine neue Zeit, auf neue Institutionen und neue mediale Formen überträgt - wobei diese medialen Formen weniger solche des Bildes denn solche des Wortes und der Schrift sind: Während das Bild (imago/effigies), das Franziskus selbst ist, von den künstlerischen Bildern (tabulae) abgesetzt wird, die das Göttliche darstellen, heißt es am Ende der Episode, das Lob der heiligen Wundmale sei in Sequenzen, Hymnen und Antiphonen verkündet worden (laudes sacrorum stigmatorum prosis et hymnis et antiphonis; LM XIII,10).

\section{V}

Bonaventura präsentiert nicht nur eine biographische Synthese, sondern auch eine mediale. In ihrem Zentrum stehen Schrift, Bild und Körper - als jene drei Formen, in denen Tradition und Gegenwärtigkeit, Substanz und Erscheinung, Materialität und Übertragung zusammentreffen. Sie sind im Text scheinbar nacheinander geschaltet: Der Weg führt von der Meditation über die Vision zur Impression, von der Benutzung des Buches über die Erscheinung des Seraphs zur Einprägung der Wundmale. Zugleich aber sind die drei Formen, Schrift, Bild und Körper, ineinander verflochten: Körperliche Praktiken (Fasten, Leiden) bereiten schon vor, was sich dann als radikale Körpererfahrung ereignen wird; Momente der Schrift kehren auch nach dem Abstieg vom La Verna wieder; die Stichworte visio, effigies und figura werden am Ende in einer direkten Anrede an den Heiligen aufgegriffen (LM XIII,10). Überdies sind alle drei paradigmatisch miteinander verbunden. Sie haben jeweils einen doppelten, nämlich sowohl allgemeinen wie spezifischen, abstrakten

22 LM XIII,5: non in tabulis lapideis vel ligneis manu figuratam artificis, sed in carneis membris descriptam digito Dei vivi. 
wie konkreten Charakter. Die Schrift ist gegeben als sacra scriptura, als selbst zweigeteilte heilige Schrift, geoffenbart, autoritativ, traditionsstiftend, unaufhörlicher Bezugspunkt der Rede. Sie ist aber auch gegeben als materielles Gebilde, als liber sacer, als das konkrete Evangelienbuch, das Franziskus bei sich hat und das ihm im Orakel die ultimative Gewissheit vermittelt, der werden zu können, der er in seinem Innersten schon ist. Das Bild ist die effigies, die Franziskus in seiner visio erscheint, ist aber auch er selbst, der er zu einer effigies wird, welche den Crucifixus auf sich, an sich und mit sich trägt (secum ferens). Der Körper ist derjenige des Engels und des Gekreuzigten, ist aber auch derjenige des Asketen, auf den sich die Zeichen übertragen.

In jedem Fall handelt es sich nicht um eine statische Form, die etwas festhält, wiedergibt oder darstellt, sondern um eine dynamische, mit und in der etwas geschieht, die in Bewegung ist, sich verwandelt und ihrerseits Verwandlung bewirkt. Schrift, Bild und Körper sind in diesem Zusammenhang nicht einfach Medien, sofern man darunter distinkte, institutionalisierte Typen der Vermittlung, Übertragung, Verbreitung, Speicherung oder Kommunikation versteht. Sie überlagern sich, ergänzen sich, stehen im Austausch. Das eigentliche Medium ist Franziskus, in dem sich seinerseits das Urmedium Christus neu verkörpert. Er ist als „Zeichen des lebendigen Gottes“ und „Zeugnis der Wahrheit" im Sinne der Zeit ein exemplar: das Bild eines Urbildes, das selbst zum Urbild wird, die perfekte Verkörperung eines Mediums, das zugleich ein anderes und sich selbst sichtbar macht. Er repräsentiert die paradoxe Kombination von Identität und Differenz, die Tautologie, die zugleich Unerhörtes mit sich bringt, die Übertragung, in der Übertragenes und Übertragendes, Verfügbarkeit und Unverfügbarkeit ineinander verschlungen sind.

\section{Literatur}

\section{Texte}

Bonaventura: Legenda maior [abgekürzt zitiert: LM). In: Analecta Franciscana 10 (1926-1941), S. 557-652 und ders.: Legenda minor. In: Analecta Franciscana 10 (1926-1941), S.655-678. Nachdruck in: Fontes Franciscani, a cura di Enrico Menestò e Stefano Brufani et al. Assisi 1995. Deutsche Übersetzung: Franziskus, Engel des sechsten Siegels. Sein Leben nach den Schriften des heiligen Bonaventura. Einführung, Übersetzung, Anmerkungen [von] Sophronius Clasen. Werl/Westf. 1962 (Franziskanische Quellenschriften 7).

\section{Forschungsliteratur}

Adnès, Pierre (1990): Art. Stigmates. In: Dictionnaire de spiritualité 14, Sp. 1211-1243.

Belting, Hans (2006): Franziskus. Der Körper als Bild. In: Bild und Körper im Mittelalter. Hg. von Kristin Marek u. a. München.

Bösch, Paul (2005): Franz von Assisi - neuer Christus. Die Geschichte einer Verklärung. Düsseldorf. Bouflet, Joachim (1996): Les stigmatisés. Paris.

Bougerol, Jacques Guy (1984): Francesco e Bonaventura: La Legenda major. Vicenza.

Brooke, Rosalind B. (2006): The Image of St. Francis. Responses to Sainthood in the Thirteenth Century. Cambridge. 
Browe, Peter (1938): Die eucharistischen Wunder des Mittelalters. Breslau (Breslauer Studien zur historischen Theologie N. S. 4).

Browe, Peter (2003): Die Eucharistie im Mittelalter. Liturgiehistorische Forschungen in kulturwissenschaftlicher Absicht. Münster (Vergessene Theologen 1).

Dalarun, Jacques (1999): François d'Assise ou le pouvoir en question. Principes et modalités du gouvernement dans l'ordre des Frères mineurs. Paris, Brüssel (Bibliothèque du moyen âge 15).

Davidson, Arnold I. (2009): Miracles of Bodily Transformation, or How St. Francis Received the Stigmata. In: Critical Inquiry 35/3, S. 451-480.

Daxelmüller, Christoph (2001): Süße Nägel der Passion. Die Geschichte der Selbstkreuzigung von Franz von Assisi bis heute. Düsseldorf.

Feld, Helmut (1994): Franziskus von Assisi und seine Bewegung. Darmstadt.

Flood, David E. (2005): Franziskus und die Offenheit der Geschichte. In: Franziskus von Assisi. Hg. von Dieter R. Bauer/Helmut Feld/Ulrich Köpf. Köln, S. 97-106.

Frugoni, Chiara (1993): Francesco e l'invenzione delle stimmate. Una storia per parole e immagini fino Bonaventura e Giotto. Turin (Saggi 780).

Kapustka, Mateusz (2010): Die Kutte. Zur medialen Verlängerung des bildgewordenen Franziskuskörpers. In: Kleidung im Bild. Zur Ikonologie dargestellter Gewandung. Hg. von Philipp Zittlsperger. Emsdetten, Berlin (Textile Studies 1), S. 33-47.

Kiening, Christian/Beil, Ulrich Johannes (2012): Urszenen des Medialen. Von Moses zu Caligari. Göttingen.

Marek, Kristin (2008): Die Körper des Königs. Zur Bildkultur der Effigies. München.

Melville, Gert/Oberste, Jörg (Hg.): Die Bettelorden im Aufbau. Beiträge zu Institutionalisierungsprozessen im mittelalterlichen Religiosentum. Münster, Hamburg, London (Vita regularis 11).

Menke, Bettine/Vinken, Barbara (Hg.) (2004): Stigmata. Poetiken der Körperinschrift. München.

Pavlick, Elizabeth-Jane (2007): “The Sanctified Senses of the Holy Man”: Bonaventure's Theology of the Body. In: Collectanea Franciscana 77, S. 541-567.

Schmucki, Octavian (1991): The Stigmata of St. Francis of Assisi: A Critical Investigation in the Light of Thirteenth-Century Sources, St. Bonaventure. NY (Franciscan Institute Publications. History series 6). 